\title{
Iterative Magnetometer Calibration
}

\author{
Joseph Sedlak \\ Email: sedlak@ai-solutions.com \\ a.i. solutions, Inc. \\ 10001 Derekwood Lane, Suite 215 \\ Lanham, MD 20706 USA
}

\begin{abstract}
This paper presents an iterative method for three-axis magnetometer (TAM) calibration that makes use of three existing utilities recently incorporated into the attitude ground support system used at NASA's Goddard Space Flight Center. The method combines attitude-independent and attitude-dependent calibration algorithms with a new spinning spacecraft Kalman filter to solve for biases, scale factors, nonorthogonal corrections to the alignment, and the orthogonal sensor alignment. The method is particularly well-suited to spin-stabilized spacecraft, but may also be useful for three-axis stabilized missions given sufficient data to provide observability.
\end{abstract}

\section{Introduction}

Recent work on the attitude ground support system (AGSS) ${ }^{1.2}$ used for mission support at the NASA Goddard Space Flight Center has included incorporation of an improved attitude-independent calibration utility for threeaxis magnetometers (TAMs) and a new Kalman filter for spin-stabilized spacecraft. A new attitude-dependent alignment estimation utility was added a few years ago. The current work describes how these three utilities have been combined to create a tool for full TAM calibration. The new method is particularly well-suited to spin-stabilized spacecraft, where such a tool had been lacking. For three-axis stabilized spacecraft having more accurate sensors (e.g., star trackers and gyros), other TAM calibration algorithms are available, but the new method described here may still be useful for a preliminary calibration prior to calibration of the fine sensors. This is valuable for missions such as Aqua and Aura that require moderate accuracy from the coarse sensors in order to acquire and identify stars before transitioning to fine-pointing mode.

Using data only from the TAM and one or more other sensors, the new calibration method determines the full set of calibration parameters except for the TAM-torquer coupling matrix. (Contamination of the TAM signal due to coupling with the magnetic torquer is not considered in this paper but is left for future work. Also, one can often avoid this issue by selecting only data when the torquers are off.) The method is motivated in part by experiments with iterative TAM calibration using UARS and EUVE mission data. ${ }^{3}$

The purpose of TAM calibration is to improve estimates of the parameters needed to convert raw measurements into body-frame observations of the instantaneous geomagnetic field. For the usual TAM sensor model, these parameters are the sensor bias and scale factor for each axis and the alignments of the axes. A coarse calibration often estimates only the biases. With sufficient data, an attitude-independent, TAM-only method can determine the biases, scale factors, and the nonorthogonal part of the alignment (a measure of the skewness of the three axes). Alonso and Shuster ${ }^{4}$ give an excellent survey of attitude-independent methods. When data from other sensors is available, the orthogonal alignment can be determined relative to those other sensors.

\section{Method}

The new algorithm combines attitude-independent and attitude-dependent estimators with a new Kalman filter designed for spinning spacecraft. Specifically:

1. Apply Alonso and Shuster's TWOSTEP method ${ }^{5}$ to determine the bias, scale factor, and nonorthogonal part of the alignment. Readjust the TAM data with these parameters.

2. Solve for an attitude history using the readjusted TAM and Sun data (or whatever other sensors are available) with the spinning spacecraft Kalman filter (SpinKF) ${ }^{6}$ for spin-stabilized missions or other attitude determination utility for three-axis stabilized missions.

3. Apply the ALIQUEST attitude-dependent alignment estimation utility ${ }^{7}$ to determine orthogonal alignments of the TAM and Sun sensor. Combine into a single TAM alignment. Readjust the TAM data.

Repeat steps 2 and 3 until no further significant changes are found in the parameters. 


\section{Test Results}

The calibration algorithm was tested with a series of simulations using parameters based on the ST5 mission. These simulations modeled the spacecraft as axially symmetric so an exact solution for the dynamics could be used. The inertia tensor was taken to be diagonal, with diagonal elements $[0.8 ; 0.8 ; 1.12] \mathrm{kg}-\mathrm{m}^{2}$. The spin rate is 26 revolutions per minute. The TAM measurement frequency is $8 \mathrm{~Hz}$. The slit Sun sensor noise is $0.18 \mathrm{deg}$, and the TAM noise is $2 \mathrm{mG}$ (corresponding roughly to $0.5 \mathrm{deg}$ error), both assumed to be white and zero-mean. The simulations did not include maneuvers, but the attitude was initialized with a nutation angle of 2 deg. For this test, one hour of data was simulated.

The TAM data was corrupted with a bias vector of $[1 ;-2 ; 3] \mathrm{mG}$ and an orthogonal misalignment with respect to the nominal TAM frame corresponding to a rotation vector of $[-2 ; 1.5 ; 1] \mathrm{deg}$. Figure 1 shows the resulting precalibration errors in the attitude estimate with respect to the truth model. The root-mean-square values of the $X$-, $Y$-, and Z-errors are $0.870,0.896$, and $1.056 \mathrm{deg}$, for which the root-sum-square (RSS) value is $1.635 \mathrm{deg}$. Figure 2 shows that the post-calibration attitude estimate errors are much smaller; the RSS value is $0.151 \mathrm{deg}$. The estimated bias vector is $[0.999 ;-2.036 ; 3.009] \mathrm{mG}$, and the estimated misalignment rotation vector is $[-2.02 ; 1.42 ; 0.89]$ deg. Additional test results are given in the paper.

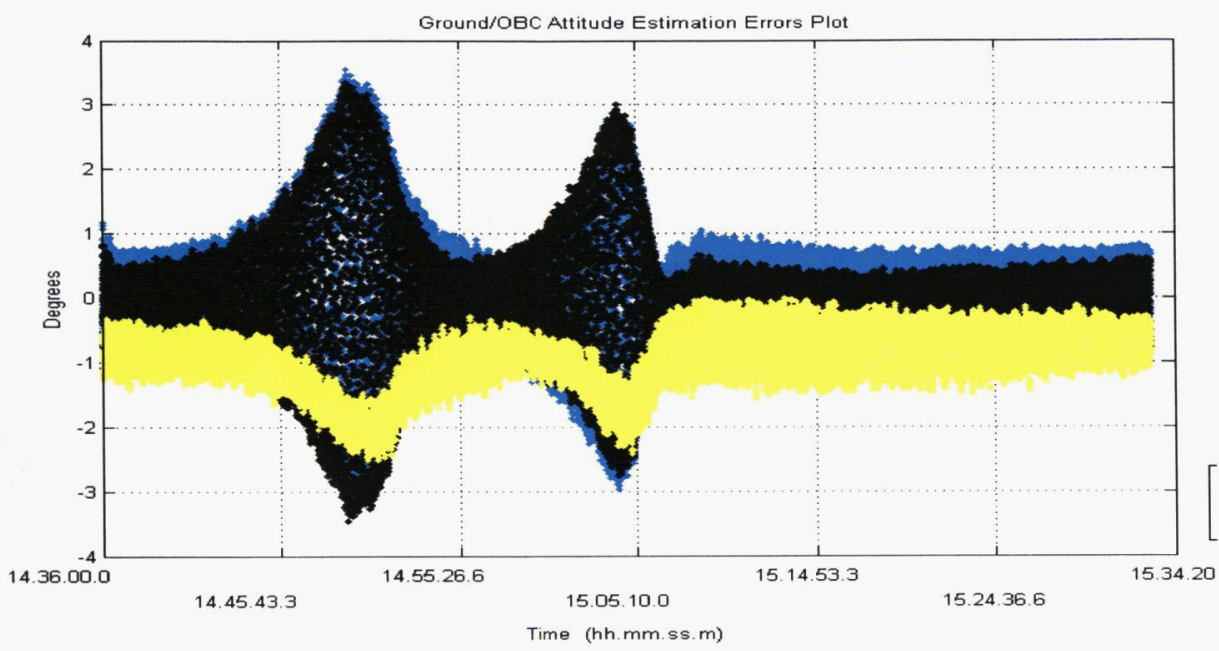

20060228.143600000

20060228.153300000

Fig. 1. Pre-calibration comparison between estimated attitude and truth model. Blue, black, and yellow curves show attitude errors as rotations about the body $X$-, $Y$-, and $Z$-axes, respectively.

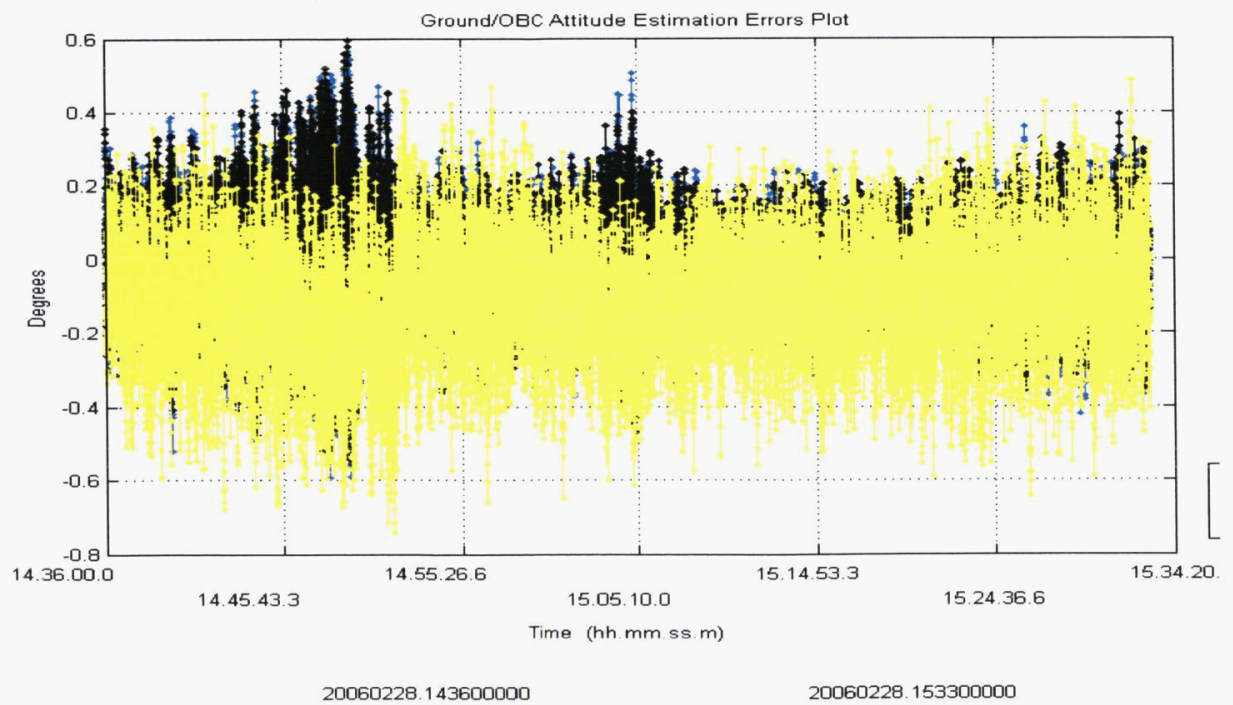

Fig. 2. Post-calibration comparison between estimated attitude and truth model. Blue, black, and yellow curves show attitude errors as rotations about the body $X$-, $Y$-, and $Z$-axes, respectively. 


\section{References}

1. Neil Ottenstein, et al., "Multi-Mission Spin-Axis Stabilized Spacecraft (MSASS) Functional Specifications," Computer Sciences Corporation technical report prepared for NASA/ GSFC, CSC/TR-90/6051R1UD0, Mar. 1993

2. Jon Landis, et al., "Multi-Mission Three-Axis Stabilized Spacecraft (MTASS) Functional Specifications, Rev. 1," Computer Sciences Corporation technical report prepared for NASAGSFC, 554-FDD-91/070R1UD0, CSC/TR-91/6071R1UD0, Sep. 1995

3. Joseph A. Hashmall, Kuen-San Liu, and Mohammad Rokni, "Accurate Spacecraft Attitudes From Magnetometer Data," Int. Symposium on Spaceflight Dynamics, CNES, Toulouse, June 1995

4. Roberto Alonso and Malcolm D. Shuster, "Attitude-Independent Magnetometer-Bias Determination: A Survey," JAS, Vol. 50, No. 4, Oct.-Dec. 2002, pp. 453-475

5. Roberto Alonso and Malcolm D. Shuster, "Complete Linear Attitude-Independent Magnetometer Calibration," JAS, Vol. 50, No. 4, Oct.-Dec. 2002, pp. 477-490

6. Joseph E. Sedlak, "Spinning Spacecraft Attitude Estimation Using Markley Variables: Filter Implementation and Results," 2005 Flight Mechanics Symposium, NASA Conference Publications NASA/CP-2005-212789, NASA/Goddard Space Flight Center, Greenbelt, MD, Oct. 2005

7. Joseph A. Hashmall and Joseph E. Sedlak, "New Attitude Sensor Alignment Calibration Algorithms," $53^{\text {rd }}$ International Astronautical Congress, Houston, TX, IAF, Oct. 2002 